\title{
Psoriasin expression is associated with survival in patients with human papillomavirus-positive base of tongue squamous cell carcinoma
}

\author{
MARK ZUPANCIC $^{1 *}$, LINNEA HAEGGBLOM ${ }^{1 *}$, DAVID LANDIN $^{2}$, \\ LINDA MARKLUND $^{2}$, TINA DALIANIS ${ }^{1 * *}$ and ANDERS NÄSMAN ${ }^{1,3 * *}$ \\ ${ }^{1}$ Department of Oncology-Pathology; ${ }^{2}$ Division of Ear, Nose and Throat Diseases, \\ Department of Clinical Science, Intervention and Technology - CLINTEC, Karolinska Institutet, 17164 Stockholm; \\ ${ }^{3}$ Department of Clinical Pathology, Karolinska University Hospital, 17177 Stockholm, Sweden
}

Received November 1, 2020; Accepted January 25, 2021

DOI: $10.3892 / \mathrm{ol} .2021 .12538$

\begin{abstract}
Patients with human papillomavirus-positive (HPV+) base of tongue squamous cell carcinomas (BOTSCC) have an improved survival compared with patients with HPV-negative BOTSCC and it has been suggested that treatment should be tailored. Before individualized treatment can be introduced, additional prognostic markers are required. A prognostic role of psoriasin has previously been demonstrated outside BOTSCC. Therefore, the present study aimed to examine psoriasin in BOTSCC, with focus on HPV+ BOTSCC, in relation to prognosis. A total of 72 BOTSCC samples were stained for psoriasin by immunohistochemistry, and the association between expression and clinical outcomes was analyzed. Patients with low psoriasin expression exhibited significantly improved overall survival (OS; $\mathrm{P}=0.001)$ and disease-free survival (DFS; $\mathrm{P}=0.007$ ), which also was observed in patients with HPV+ BOTSCC (OS, P<0.001; DFS, $\mathrm{P}=0.02$ ). Furthermore, psoriasin was a significant prognostic
\end{abstract}

Correspondence to: Professor Tina Dalianis or Dr Anders Näsman, Department of Oncology-Pathology, Karolinska Institutet, Bioclinicum J6:20, 17164 Stockholm, Sweden

E-mail: tina.dalianis@ki.se

E-mail: anders.nasman@ki.se

\section{${ }^{* * * * *}$ Contributed equally}

Abbreviations: BOTSCC, base of tongue squamous cell carcinoma; DFS, disease-free survival; FFPE, formalinfixed paraffin-embedded; HNSCC, head and neck squamous cell carcinoma; HPV, human papillomavirus; HPV-, human papillomavirus-negative; $\mathrm{HPV}+$, human papillomavirus-positive; IHC, immunohistochemistry; OPSCC, oropharyngeal squamous cell carcinoma; OS, overall survival

Key words: oropharyngeal cancer, prognosis, psoriasin, s100A7, human papillomavirus factor in univariable and multivariable analyses. In conclusion, psoriasin could be used as a prognostic marker in HPV+ BOTSCC.

\section{Introduction}

Human papillomavirus positive (HPV+) oropharyngeal squamous cell carcinomas (OPSCC) are increasing in incidence and patients with HPV+ OPSCC have a much better clinical outcome, as compared to patients with HPV negative (HPV-) OPSCC. Therefore, today, HPV+ and HPV- OPSCC are considered as two separate entities and are staged separately according the new AJCC/IUCC staging manual (TNM-8). Over the last years, it has also been debated if oncological treatment of patients with HPV+ OPSCC can be tapered, but previous attempts have not been successful (1). Notably, however, accumulated recent data advocate that HPV+ OPSCC should be divided into its sub-sites, when categorizing by HPV status and prognosis, more specifically tonsillar squamous cell carcinoma (TSCC), base of tongue squamous cell carcinoma (BOTSCC) and squamous cell carcinoma of the soft palate and the pharyngeal walls (other OPSCC) (2). Data from others and us clearly indicate that patients with HPV+ TSCC/BOTSCC have a better survival than patients with HPV- TSCC/BOTSCC, but this survival benefit of having HPV was not observed in patients with other OPSCC (3-7). Nevertheless, although the survival in general is favorable in patients with HPV+ TSCC/BOTSCC, prognostic markers are still needed to identify those few patients with a poor clinical outcome, before treatment can be tapered (8).

Psoriasin, or s100A7, is a protein part of the S100 family containing calcium-binding motifs and is an important cell mediator for e.g. cell survival and maturation. Increased expression of the protein has been reported in malignant and premalignant lesions and overexpression has also been correlated with clinical outcome (9-14). In a previous study by Tripathi et al (15), the expression of psoriasin was correlated to a worse prognosis in patients with head and neck carcinomas (HNSCC), but neither HPV status nor sub-site was considered in that study. 

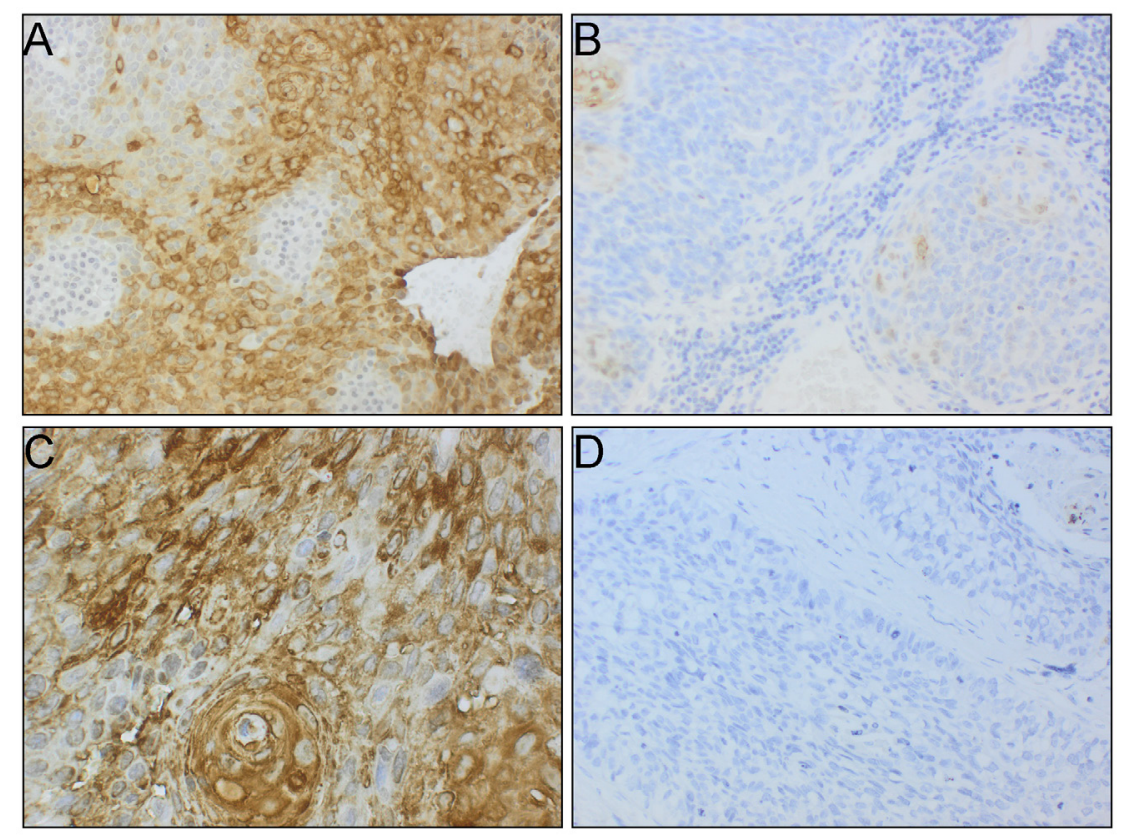

Figure 1. Representative images of psoriasin immunohistochemistry staining. Magnification, x400. (A) High expression in HPV+ BOTSCC. (B) Low expression in HPV+ BOTSCC. (C) High expression in HPV- BOTSCC. (D) Low expression in HPV- BOTSCC. BOTSCC, base of tongue squamous cell carcinoma; HPV, human papillomavirus; HPV+, HPV DNA-positive; HPV-, HPV DNA-negative.

Hence, in this study, we wanted to examine the previously reported prognostic significance of psoriasin staining obtained in a heterogenous HNSCC patient cohort, in a homogenous pilot cohort of only BOTSCC. This specific cohort had also previously been tested for the presence or absence of HPV DNA and p16 overexpression $(16,17)$ and was therefore useful for examining psoriasin expression in correlation to HPV status, as well as to clinical outcome.

\section{Materials and methods}

Patients and tumors. Patients diagnosed with BOTSCC 2000-2007 in the County of Stockholm, previously tested for HPV DNA by Luminex Multiplex PCR and $\mathrm{p} 16^{\mathrm{INK} 4 \mathrm{a}}$ (p16) overexpression ( $>70 \%$ cytoplasmatic and nuclear tumor expression) by immunohistochemistry, were identified $(16,17)$. Available formalin-fixed paraffin-embedded (FFPE) pretreatment biopsies (obtained through an ENT forceps biopsy) were collected from the Department of Clinical Pathology, Karolinska University Hospital. Patient data were collected from patients records. The study was conducted according to ethical permissions 2009/1278-31/4 and 2017/1035-31/2, Karolinska Institutet.

Immunohistochemistry and staining evaluation. Tumor sections $(4 \mu \mathrm{m})$ were cut and deparaffinized in Xylene and rehydrated in graded ethanol. Heat-induced antigen retrieval was performed with citric acid buffer (pH6) using a microwave oven for $10 \mathrm{~min}$, which was followed by $\mathrm{H}_{2} \mathrm{O}_{2}$ treatment in order to block endogenous peroxidase. The slides were then incubated with horse serum, followed by incubation with primary antibody (mouse mAb S100A7 dilution 1:100, clone 47C1068; Santa Cruz Biotechnology) overnight. Secondary anti-mouse antibody (1:200; Vector Laboratories, Inc.) was then added followed by the ABC kit (Vectastain; Vector
Laboratories, Inc.). The staining was developed in DAB followed by hematoxylin counterstaining. The staining was evaluated by three researchers blinded for clinical outcome (LH, DL and AN). The percentage of positive tumor cells per total tumor cells was evaluated for each section and a cut-off value of $30 \%$ (more specifically, the proportion of immunostained cells, irrespective of whether the staining was cytoplasmic or nuclear) was applied as previously described, and since we here wanted to compare out data to such studies by others (15).

Statistical analysis. Differences in continuous and categorical variables were assessed with double-sided t-test and Chi-square test, respectively. Three-year overall survival (3-year OS) was defined as days from diagnosis until death. All patients were censored after three years. Three-year disease-free survival (3-year DFS) was defined as time from diagnosis until a relapse in disease. Patients that never became tumor free were censored at day 0 . All patients were censored after 3 years. Differences in survival were estimated with the log-rank test and visualized with the Kaplan-Meier method. The Cox proportional hazards regression analysis was used for the calculation of hazard ratios (HR) with 95\% confidence intervals $(95 \% \mathrm{CI})$ in the univariable and multivariable analysis. Besides S100A7 expression, established prognostic markers in OPSCC (age, TNM-status, smoking status and treatment) were included. P-values $<0.05$ were considered significant. All calculations and analyses were performed using IBM SPSS Statistics, (version 25.0; IBM. Corp.)

\section{Results}

Patients, tumors and psoriasin expression. In total, 76 patients diagnosed with BOTSCC between 2000-2007 
Table I. Patient and tumor characteristics.

\begin{tabular}{|c|c|c|c|c|}
\hline Characteristics & Low psoriasin expression & High psoriasin expression & All patients & P-value \\
\hline Age at diagnosis, years (mean) & 62 & 60 & 61 & 0.5 \\
\hline \multicolumn{5}{|l|}{ Sex, n $(\%)$} \\
\hline Female & $19(35)$ & $4(22)$ & $23(32)$ & \multirow[t]{2}{*}{0.4} \\
\hline Male & $35(65)$ & $14(78)$ & $49(68)$ & \\
\hline \multicolumn{5}{|l|}{ Stage (TNM-8), n (\%) } \\
\hline I & $21(39)$ & $6(33)$ & $27(38)$ & \multirow[t]{4}{*}{0.4} \\
\hline II & $11(20)$ & $2(11)$ & $13(18)$ & \\
\hline III & $14(26)$ & $4(22)$ & $18(25)$ & \\
\hline IV & $8(15)$ & $6(33)$ & $14(19)$ & \\
\hline \multicolumn{5}{|l|}{ Treatment $^{\mathrm{a}}, \mathrm{n}(\%)$} \\
\hline RT & $25(46)$ & $14(78)$ & $39(54)$ & \multirow[t]{2}{*}{0.03} \\
\hline CRT & $29(54)$ & $4(22)$ & $33(46)$ & \\
\hline \multicolumn{5}{|l|}{ Radiotherapy $^{\mathrm{b}}, \mathrm{n}(\%)$} \\
\hline Conventional & $39(72)$ & $12(67)$ & $51(71)$ & \multirow[t]{2}{*}{0.8} \\
\hline Accelerated & $15(18)$ & $6(33)$ & $21(29)$ & \\
\hline \multicolumn{5}{|l|}{ Cetuximab treatment ${ }^{\mathrm{c}}, \mathrm{n}(\%)$} \\
\hline No & $52(96)$ & $18(100)$ & $70(97)$ & \multirow[t]{2}{*}{$>0.9$} \\
\hline Yes & $2(4)$ & $0(0)$ & $2(3)$ & \\
\hline \multicolumn{5}{|l|}{ Current smoker ${ }^{\mathrm{d}}, \mathrm{n}(\%)$} \\
\hline No & $42(78)$ & $8(44)$ & $50(69)$ & \multirow[t]{2}{*}{0.02} \\
\hline Yes & $12(22)$ & $10(56)$ & $22(31)$ & \\
\hline \multicolumn{5}{|l|}{ HPV DNA status ${ }^{\mathrm{e}}, \mathrm{n}(\%)$} \\
\hline Negative & $6(11)$ & $10(56)$ & $16(22)$ & \multirow[t]{2}{*}{$<0.001$} \\
\hline Positive & $48(89)$ & $8(44)$ & $56(78)$ & \\
\hline \multicolumn{5}{|l|}{ p16 upregulation ${ }^{\mathrm{e}}, \mathrm{n}(\%)$} \\
\hline Negative & $9(17)$ & $11(61)$ & $20(28)$ & \multirow[t]{2}{*}{$<0.001$} \\
\hline Positive & $45(83)$ & $7(39)$ & $52(72)$ & \\
\hline \multicolumn{5}{|c|}{ HPV DNA and p16 upregulation ${ }^{\mathrm{e}}, \mathrm{n}(\%)$} \\
\hline Negative & $10(19)$ & $10(56)$ & $20(28)$ & \multirow[t]{2}{*}{0.005} \\
\hline Positive & $44(81)$ & $8(44)$ & $52(72)$ & \\
\hline
\end{tabular}

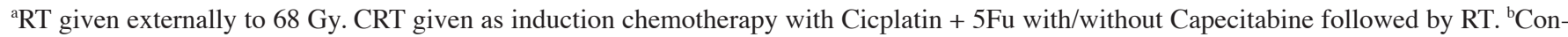
ventional RT given as $2.0 \mathrm{~Gy} /$ day in 6.5-7 weeks; total dose $68 \mathrm{~Gy}$. Accelerated RT given as $1.1+2.0$ Gy per day in 4.5 weeks; total dose 68 Gy. ${ }^{\mathrm{c}}$ Cetuximab given concomitant. dSmoking status was defined as smoker/non-smoker upon diagnosis. ${ }^{\mathrm{e}}$ Data obtained from previous study (7). RT, radiotherapy; CRT, chemo-radiotherapy; HPV, human papillomavirus; p16, p16INK4a; ns, not significant.

in Stockholm, treated with curative intent and with tumors tested for possible presence of HPV DNA (HPV DNA positive by PCR) and overexpression of p16 were identified from previous publications $(16,17)$. Tumor slides, available from 72 of these 76 patients, were subsequently stained for S100A7 (psoriasin) expression. Patients and tumor characteristics of these 72 patients are depicted in Table I.

Psoriasin expression varied greatly in the invasive tumor areas, ranging from no staining to $100 \%$ stained tumor cells. The majority of tumors $(n=36)$ had no (absent) psoriasin staining $(0 \%)$ in invasive BOTSCC. When a cut-off of $30 \%$ positivity was applied [as previously suggested by others (15)], 18 tumors were positive in their invasive tumor component and were defined as having high psoriasin expression
(Fig. 1A and C). Remaining patients $(n=18)$ had low psoriasin expression in their invasive BOTSCC (Fig. 1B and D), and were grouped with the patients with tumors not expressing psoriasin (low psoriasin expression group).

Patients with BOTSCC and low psoriasin expression were significantly more often treated with chemo-radiotherapy, were significantly more often not a current smoker, and their tumors were significantly more often HPV+ (Table I). Psoriasin expression was not correlated to histological grade (data not shown).

Psoriasin expression and correlation to prognosis in patients with HPV+ and HPV-BOTSCC. Patients with HPV+ BOTSCC (presence of HPV DNA) and having high psoriasin 
Table II. Univariable and multivariable analysis of OS and DFS in patients with human papillomavirus DNA-positive base of tongue squamous cell carcinoma.

\begin{tabular}{|c|c|c|c|c|c|c|c|c|c|c|c|c|}
\hline \multirow[b]{3}{*}{ Variables } & \multicolumn{6}{|c|}{ OS } & \multicolumn{6}{|c|}{ DFS } \\
\hline & \multicolumn{3}{|c|}{ Univariable } & \multicolumn{3}{|c|}{ Multivariable } & \multicolumn{3}{|c|}{ Univariable } & \multicolumn{3}{|c|}{ Multivariable } \\
\hline & $\mathrm{HR}$ & $95 \% \mathrm{CI}$ & P-value & HR & $95 \% \mathrm{CI}$ & $\mathrm{P}$-value & HR & $95 \% \mathrm{CI}$ & $\mathrm{P}$-value & HR & $95 \% \mathrm{CI}$ & $\mathrm{P}$-value \\
\hline \multicolumn{13}{|c|}{ TNM-8 stage } \\
\hline I and II & 1 & & & 1 & & & 1 & & & 1 & & \\
\hline III and IV & 4.7 & $0.94-23$ & 0.06 & 3.4 & $0.60-19$ & 0.2 & 0.82 & $0.15-4.5$ & 0.8 & 0.15 & $0.010-2.3$ & 0.2 \\
\hline $\mathrm{Age}^{\mathrm{a}}$ & 1.0 & $0.96-1.1$ & 0.5 & 1.1 & $0.97-1.2$ & 0.2 & 1.1 & $0.97-1.2$ & 0.2 & 1.3 & $1.0-1.6$ & 0.03 \\
\hline \multicolumn{13}{|l|}{ Treatment } \\
\hline CRT & 1 & & & 1 & & & 1 & & & 1 & & \\
\hline RT & 0.59 & $0.14-2.5$ & 0.5 & 1.1 & $0.23-5.0$ & 0.9 & 0.46 & $0.084-2.5$ & 0.4 & 0.28 & $0.022-3.6$ & 0.3 \\
\hline \multicolumn{13}{|c|}{ Current smoker } \\
\hline Yes & 1 & & & 1 & & & 1 & & & 1 & & \\
\hline No & 0.32 & $0.075-1.4$ & 0.1 & 0.45 & $0.093-2.2$ & 0.3 & 0.17 & $0.034-0.84$ & 0.03 & 0.0060 & $0-0.21$ & 0.005 \\
\hline \multicolumn{13}{|l|}{ Psoriasin } \\
\hline Low & 1 & & & 1 & & & 1 & & & 1 & & \\
\hline High & 8.3 & $2.1-33$ & 0.003 & 13 & $2.0-88$ & 0.007 & 6.0 & $1.1-33$ & 0.04 & 230 & $3.8-14000$ & 0.01 \\
\hline
\end{tabular}

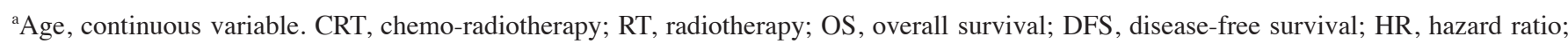
95\% CI, 95\% confidence interval.
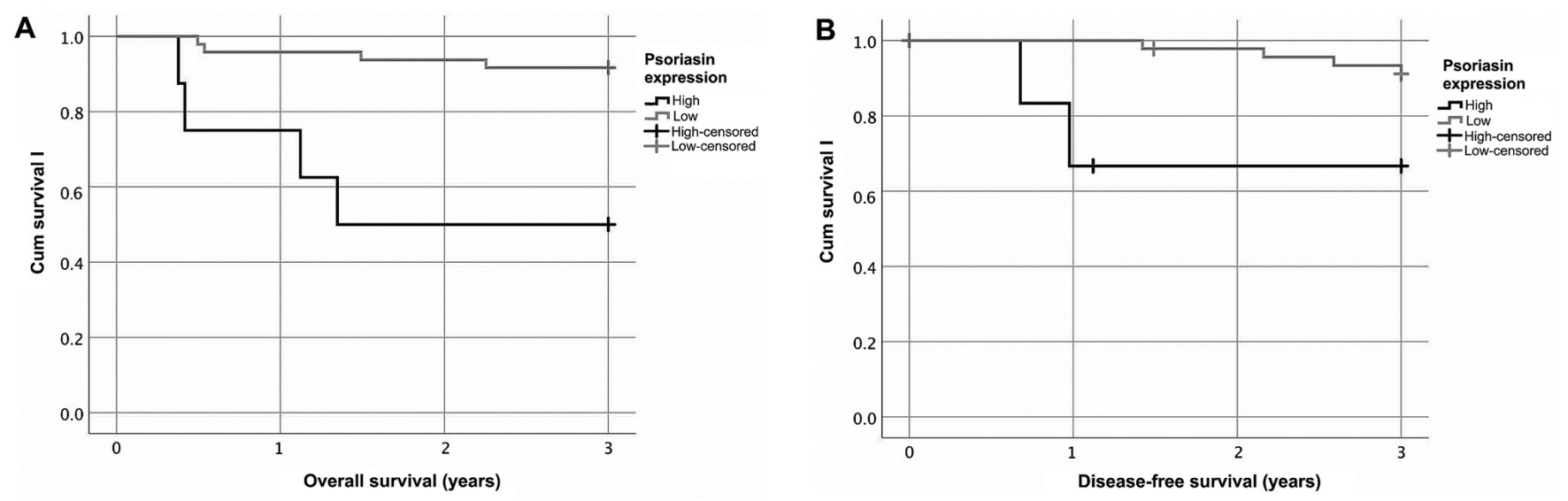

Figure 2. Kaplan-Meier curves of (A) OS and (B) DFS in patients with HPV+ BOTSCC. Tumors were stratified into a high ( $\geq 30 \%$; 'high') and a low ( $<30 \%$; 'low') fraction of psoriasin-positive tumor cell expression in immunohistochemistry. High tumor cell expression of psoriasin was significantly associated with $(\mathrm{A})$ a worse OS $(\mathrm{P}<0.001)$ and $(\mathrm{B})$ a worse DFS $(\mathrm{P}=0.02)$ in patients with HPV+ BOTSCC. The OS in patients with HPV+ BOTSCC was $85.7 \%(91.7 \%$ in patients with low psoriasin expression vs. 50\% in patients with high psoriasin expression). Similarly, the DFS in patients with HPV+ BOTSCC was $89.3 \%$ (91.7\% in patients with low psoriasin expression vs. $66.7 \%$ in patients with high psoriasin expression). BOTSCC, base of tongue squamous cell carcinoma; cum, cumulative; DFS, disease-free survival; HPV, human papillomavirus; HPV+, HPV DNA-positive; OS, overall survival.

expression ( $\geq 30 \%$ ) had a significantly worse overall survival (OS) and disease-free survival (DFS), as compared to patients with HPV+ BOTSCC and having a low psoriasin expression (log rank test: OS $\mathrm{P}<0.001$; DFS $\mathrm{P}=0.02$; Fig. 2). Similar results were obtained when HPV status was defined as p16 overexpression ( $>70 \%$ p16 positive tumor cells) alone (log rank test: $\mathrm{OS} \mathrm{P}<0.001$; DFS $\mathrm{P}=0.02$; data not shown) or when HPV DNA positive combined with over expression of p16 (log rank test: OS $\mathrm{P}<0.001$; DFS $\mathrm{P}=0.03$; data not shown) was tested. Moreover, high psoriasin expression was independently correlated to a worse OS and DFS both in uni- and multivariable analysis, including previously known prognostic factors (Table II).

In contrast, however, no differences in survival were observed in patients with HPV DNA negative BOTSCC between low and high psoriasin expression (log rank test: OS $\mathrm{P}=0.8$; DFS $\mathrm{P}=0.9$; data not shown). Similarly, no differences were identified when HPV negative status was defined as no 
p16 overexpression or as absence of HPV DNA in combination with no p16 overexpression (data not shown).

Finally, low psoriasin expression as compared to having a high psoriasin expression in BOTSCC, irrespective of HPV DNA or p16 status, correlated significantly to a better 3-year OS and DFS in these patients $(\mathrm{P}=0.001$ and $\mathrm{P}=0.007$, respectively; data not shown).

\section{Discussion}

In this short study, we demonstrate a prognostic role of psoriasin in a pathological homogenous cohort of only BOTSCC, and more specifically in HPV associated BOTSCC, suggesting that psoriasin could potentially be used as a prognostic marker also in HPV associated OPSCC.

Numerous previous studies have established the prognostic role of HPV in OPSCC and there is a discussion if and how treatment can be tapered in patients with HPV+ OPSCC $(1,18)$. Nevertheless, before such de-escalation may be introduced, additional prognostic markers are needed to stratify these patients, in order to avoid undertreatment. Many such markers have been proposed in HPV+ OPSCC, but few have been validated in separate cohorts/studies (8). In this study, we confirm the previously reported prognostic role of psoriasin expression in head and neck cancer (15) in a homogenous cohort of BOTSCC, supporting that psoriasin expression correlates to an unfavorable clinical outcome, especially in patients with HPV+ BOTSCC.

Furthermore, recent data indicate, several prognostic markers could be assessed together in different prognostic algorithms in order to better separate patients with HPV+ OPSCC and a favorable clinical outcome from those with HPV+ OPSCC and a poor clinical outcome (19). It is possible that psoriasin also should be included in such algorithm.

There are some limitations in this study. No prognostic effect of psoriasin expression could be observed in patients with HPV DNA negative BOTSCC, however only 16 such patients were included, and data on the HPV DNA negative BOTSCC group should therefore be interpreted with great caution. In addition, in this pilot study, patients with TSCC and otherOPSCC were not included. In future studies, to assess the role of psoriasin expression in OPSCC and its subgroups, it would be beneficial to include larger patient groups, including more patients with HPV associated and non-associated BOTSCC, TSCC and otherOPSCC. Larger, studies would also allow for studies of a potential correlation of the presence of high psoriasin expression and smoking as well as other characteristics of the patients and their tumors.

Finally, one could argue, that it could be possible to use other cut-off values for psoriasin positivity, rather than a cut-off of $30 \%$, used here and by others (15). In this specific patient material, an optimal cut-off value according to Youden's index would be $15 \%$ for OS and $7.5 \%$ for DFS (data not shown). However, new cut-off levels for evaluating psoriasin positivity and survival, would also be needed to be validated in separate and larger cohorts. Therefore, the present cut-off should be regarded as a validation of a similar previously published cut-off (15).
Nevertheless, increased expression of S100A7 protein has been described in various cancer types (e.g. breast, bladder and head and neck carcinomas) and often with a correlation to poor clinical outcome (9-14,20). In addition, S100A7 has been shown to be functionally linked to oncogenic properties in oral SCCs, as depletion of this gene inhibited cell growth, invasion and migration (21). A handful of these prognostic studies have utilized IHC to quantify psoriasin expression. However, different quantification approaches and different cut-off levels for psoriasin expression have been used $(10,14,15)$. For this reason, we here applied a cut-off value previously used in HNSCC. Moreover, to our knowledge, the vast majorities of studies examining psoriasin expression in correlation to prognosis have shown a poor prognostic value of high psoriasin expression. However, in a study by Tiveron et al (22), where psoriasin expression was analyzed in correlation to prognosis in laryngeal carcinoma, an increased expression did not correlate to prognosis. Therefore, still, more studies are needed to verify the prognostic role of psoriasin. Moreover, it may be possible, that examining possible amplifications of mutations or methylation of the S100A7 gene in cases where psoriasin expression is correlated to poor prognosis could give more information. Nevertheless, interestingly is that it seems that psoriasin more often is expressed in in situ tumor component as compared to invasive tumor $(14,23)$, which also applies for HPV+ OPSCC (24). In line with that assumption, only a fraction of our invasive tumors showed high psoriasin expression.

Taken together, this short report confirms a prognostic role of psoriasin and the results imply that psoriasin may have a prognostic effect in HPV+ BOTSCC. In order to identify patients with HPV+ BOTSCC and a favorable clinical outcome with high sensitivity and specificity, it is possible that psoriasin should be included in such prognostic algorithm.

In conclusion, high psoriasin expression was here shown as an independent poor prognostic factor in a homogenous cohort of patients with HPV+ BOTSCC.

\section{Acknowledgements}

The authors would like to thank Mrs. Anna Malmerfelt (Department of Oncology-Pathology, Karolinska Institutet, Stockholm, Sweden) for technical assistance.

\section{Funding}

The present study was supported by Åke Wibergs Stiftelse, Jeanssons Stiftelser, Tore Nilsons Stiftelse för Medicinsk Forskning, Stiftelsen Tornspiran, Magnus Bergvalls Stiftelse, Stockholms Läns Landsting (SLL; grant no. FOUI954801), Karolinska Institutet, The Swedish Cancer Foundation (grant nos. $200704 \mathrm{Pj}$ and $200778 \mathrm{Pj}$ ) and Cancer och Allergifonden (grant nos. 194 and 10127). The funding bodies had no role in the study design, data collection, analysis, interpretation of data or in writing the manuscript.

\section{Availability of data and material}

All data generated or analyzed during this study are included in this published article. 


\section{Authors' contributions}

MZ, LH, TD and AN formulated the research question and came up with the study design. Sample selection and collection was performed by MZ, LH, LM and DL. LH and MZ performed immunohistochemistry (IHC). LH, DL and AN evaluated the IHC staining. LM and DL collected information from the patient case reports regarding response to treatment, clinical performance and survival. All raw data has been assessed by MZ, LM, TD and AN to ensure its legitimacy. MZ, LM, TD and AN analyzed, summarized and interpreted the data and wrote the manuscript, which was revised and approved by all co-authors. All authors read and approved the final manuscript.

\section{Ethics approval and consent to participate}

The present study, including patient information and consent, was conducted according to ethical permissions 2009/127831/4 and 2017/1035-31/2 from the Ethics Committee at Karolinska Institute, Stockholm, Sweden.

\section{Patient consent for publication}

Not applicable.

\section{Competing interests}

The authors declare that they have no competing interests.

\section{References}

1. Näsman A, Du J and Dalianis T: A global epidemic increase of an HPV-induced tonsil and tongue base cancer - potential benefit from a pan-gender use of HPV vaccine. J Intern Med 287: $134-152,2020$

2. Haeggblom L, Ramqvist T, Tommasino M, Dalianis T and Näsman A: Time to change perspectives on HPV in oropharyngeal cancer. A systematic review of HPV prevalence per oropharyngeal sub-site the last 3 years. Papillomavirus Res 4: 1-11, 2017.

3. Gelwan E, Malm IJ, Khararjian A, Fakhry C, Bishop JA and Westra WH: Nonuniform distribution of high-risk human papillomavirus in squamous cell carcinomas of the oropharynx: rethinking the anatomic boundaries of oral and oropharyngeal carcinoma from an oncologic HPV perspective. Am J Surg Pathol 41: 1722-1728, 2017.

4. Ljøkjel B, Lybak S, Haave H, Olofsson J, Vintermyr OK and Aarstad HJ: The impact of HPV infection on survival in a geographically defined cohort of oropharynx squamous cel carcinoma (OPSCC) patients in whom surgical treatment has been one main treatment. Acta Otolaryngol 134: 636-645, 2014.

5. Marklund L, Näsman A, Ramqvist T, Dalianis T, MunckWikland E and Hammarstedt L: Prevalence of human papillomavirus and survival in oropharyngeal cancer other than tonsil or base of tongue cancer. Cancer Med 1: 82-88, 2012.

6. Tham T, Wotman M, Roche A, Kraus D and Costantino P: The prognostic effect of anatomic subsite in HPV-positive oropharyngeal squamous cell carcinoma. Am J Otolaryngol 40: $567-572,2019$

7. Marklund L,HolzhauserS, de Flon C,Zupancic M,LandinD,Kolev A, Haeggblom L, Munck-Wikland E, Hammarstedt-Nordenvall L, Dalianis T, et al: Survival of patients with oropharyngeal squamous cell carcinomas (OPSCC) in relation to TNM 8 - Risk of incorrect downstaging of HPV-mediated non-tonsillar, non-base of tongue carcinomas. Eur J Cancer 139: 192-200, 2020.

8. Näsman A, Bersani C, Lindquist D, Du J, Ramqvist T and Dalianis T: Human papillomavirus and potentially relevant biomarkers in tonsillar and base of tongue squamous cell carcinoma. Anticancer Res 37: 5319-5328, 2017.
9. Ye L, Sun PH, Martin TA, Sanders AJ, Mason MD and Jiang WG: Psoriasin (S100A7) is a positive regulator of survival and invasion of prostate cancer cells. Urol Oncol 31: 1576-1583, 2013.

10. Liu J, Zhao Z, Sun Z, Liu C, Cheng X, Ruge F, Yang Y, Jiang WG and Ye L: Increased expression of psoriasin is correlated with poor prognosis of bladder transitional cell carcinoma by promoting invasion and proliferation. Oncol Rep 43: 562-570, 2020.

11. Hattinger E, Zwicker S, Ruzicka T, Yuspa SH and Wolf R: Opposing functions of psoriasin (S100A7) and koebnerisin (S100A15) in epithelial carcinogenesis. Curr Opin Pharmacol 13: 588-594, 2013.

12. Liu Y, Bunston C, Hodson N, Resaul J, Sun PH, Cai S, Chen G, Gu Y, Satherley LK, Bosanquet DC, et al: Psoriasin promotes invasion, aggregation and survival of pancreatic cancer cells; association with disease progression. Int J Oncol 50: 1491-1500, 2017.

13. Al-Haddad S, Zhang Z, Leygue E, Snell L, Huang A, Niu Y, Hiller-Hitchcock T, Hole K, Murphy LC and Watson PH: Psoriasin (S100A7) expression and invasive breast cancer. Am J Pathol 155: 2057-2066, 1999.

14. Emberley ED, Murphy LC and Watson PH: S100A7 and the progression of breast cancer. Breast Cancer Res 6: 153-159, 2004.

15. Tripathi SC, Matta A, Kaur J, Grigull J, Chauhan SS, Thakar A, Shukla NK, Duggal R, DattaGupta S, Ralhan R, et al: Nuclear S100A7 is associated with poor prognosis in head and neck cancer. PLoS One 5: e11939, 2010.

16. Attner P, Du J, Näsman A, Hammarstedt L, Ramqvist T, Lindholm J, Marklund L, Dalianis T and Munck-Wikland E: The role of human papillomavirus in the increased incidence of base of tongue cancer. Int J Cancer 126: 2879-2884, 2010.

17. Näsman A, Andersson E, Marklund L, Tertipis N, HammarstedtNordenvall L, Attner P, Nyberg T, Masucci GV, Munck-Wikland E, Ramqvist T, et al: HLA class I and II expression in oropharyngeal squamous cell carcinoma in relation to tumor HPV status and clinical outcome. PLoS One 8: e77025, 2013.

18. Ang KK, Harris J, Wheeler R, Weber R, Rosenthal DI, Nguyen-Tân PF, Westra WH, Chung CH, Jordan RC, Lu C, et al: Human papillomavirus and survival of patients with oropharyngeal cancer. N Engl J Med 363: 24-35, 2010.

19. Tertipis N, Hammar U, Näsman A, Vlastos A, Nordfors C, Grün N, Ährlund-Richter A, Sivars L, Haeggblom L, Marklund L, et al: A model for predicting clinical outcome in patients with human papillomavirus-positive tonsillar and base of tongue cancer. Eur J Cancer 51: 1580-1587, 2015

20. Mayama A, Takagi K, Suzuki H, Sato A, Onodera Y, Miki Y, Sakurai M, Watanabe T, Sakamoto K, Yoshida R, et al: OLFM4, LY6D and S100A7 as potent markers for distant metastasis in estrogen receptor-positive breast carcinoma. Cancer Sci 109: 3350-3359, 2018

21. Dey KK, Bharti R, Dey G, Pal I, Rajesh Y, Chavan S, Das S, Das CK, Jena BC, Halder P, et al: S100A7 has an oncogenic role in oral squamous cell carcinoma by activating p38/MAPK and RAB2A signaling pathway. Cancer Gene Ther 23: 382-391, 2016.

22. Tiveron RC, de Freitas LC, Figueiredo DL, Serafini LN, Mamede RC and Zago MA: Expression of calcium binding protein S100 A7 (psoriasin) in laryngeal carcinoma. Rev Bras Otorrinolaringol (Engl Ed) 78: 59-65, 2012.

23. Alowami S, Qing G, Emberley E, Snell L and Watson PH: Psoriasin (S100A7) expression is altered during skin tumorigenesis. BMC Dermatol 3: 1, 2003.

24. Haeggblom L, Ährlund-Richter A, Mirzaie L, Farrajota Neves da Silva P, Ursu RG, Ramqvist T and Näsman A: Differences in gene expression between high-grade dysplasia and invasive HPV+ and HPV- tonsillar and base of tongue cancer. Cancer Med 8: 6221$6232,2019$.

This work is licensed under a Creative Commons Attribution-NonCommercial-NoDerivatives 4.0 International (CC BY-NC-ND 4.0) License. 